\title{
Nutrition Knowledge, Attitudes, and Confidence of Australian General Practice Registrars
}

\author{
Caryl A. Nowson and Stella L. O'Connell \\ Centre for Physical Activity and Nutrition Research, School of Exercise and Nutrition Sciences, Deakin University, \\ Waurn Ponds Campus, Locked Bag 20000, Geelong, VIC 3220, Australia
}

Correspondence should be addressed to Caryl A. Nowson; caryl.nowson@deakin.edu.au

Received 4 December 2014; Revised 15 April 2015; Accepted 15 April 2015

Academic Editor: Friedrich Paulsen

Copyright ( 2015 C. A. Nowson and S. L. O'Connell. This is an open access article distributed under the Creative Commons Attribution License, which permits unrestricted use, distribution, and reproduction in any medium, provided the original work is properly cited.

\begin{abstract}
Nutrition knowledge, attitudes, and confidence were assessed in General Practice Registrars (GPRs) throughout Australia. Of approximately 6,000 GPRs invited to complete a nutrition survey, 93 respondents (2\%) completed the online survey, with 89 (20 males, 69 females) providing demographic and educational information. Fifty-one percent had graduated from medical school within the last two years. From a list of 11 dietary strategies to reduce cardiovascular risk, respondents selected weight loss $(84 \%)$, reducing saturated fats (90\%), a maximum of two alcoholic drinks/day (82\%), and increasing vegetables (83\%) as "highly appropriate" strategies, with only $51 \%$ indicating that salt reduction was "highly appropriate." Two-thirds of registrars felt "moderately" (51\%) or "very" confident (16\%) providing nutrition advice. Most of them (84\%) recalled receiving information during training, but only $34 \%$ recalled having to demonstrate nutritional knowledge. The results indicate that this group of Australian GPRs understood most of the key dietary recommendations for reducing cardiovascular risk but lacked consensus regarding the recommendation to reduce salt intake and expressed mixed levels of confidence in providing nutritional advice. Appropriate nutrition education before and after graduation is recommended for GPRs to ensure the development of skills and confidence to support patients to make healthy dietary choices and help prevent chronic diseases.
\end{abstract}

\section{Introduction}

Nutrition is vital to the maintenance of health and the prevention of disease. Inappropriate nutrition management contributes to the development and progression of chronic diseases, which often require longer hospitalization, leading to increased health care costs [1]. General Practice Practitioners (GPs) provide coordinated holistic health care to individuals and families in their communities. In Australia, approximately 4 years of training following graduation from medicine is required for GPs, two years in hospitals and two years in GP practice under supervision. As a medical practitioner who has received specialist training in the area of general practice, an Australian GP has undergone similar training to a US "family physician."

GPs are often the first point of call and in remote areas may be the only possible point of call, for individuals with chronic diseases where nutrition modifications are effective interventions. If doctors are equipped with the knowledge and skills to assess and address nutrition risks, they can assist in the prevention of disease, deterioration of disease, and optimal disease management $[2,3]$.

The Australian Medical Council (AMC) states that medical practitioners need to have appropriate knowledge and skills in identifying nutrition issues for patients in order to prevent and treat common chronic disease [4]. However, doctors around the world, including Australia, have been found to be ill-equipped to identify and appropriately manage nutrition-related issues of patients, whether inpatients or those living in residential care or the community [5-7]. The most common cause of insufficient nutrition practice that has been identified is lack of nutrition knowledge [3, 8, 9]. Results from a recent survey indicated that there was no consistent integration of nutrition knowledge and skills across different medical courses and that the assessment of nutrition knowledge and skills varies significantly among 
universities, with nutrition education being highly dependent on current staff interest [9].

General Practice Registrars Australia (GPRA) is the "peak...body" for prevocational doctors and GP registrars in Australia, providing nonmedical information, training, and representation for approximately $70 \%$ of GP registrars in the country who are members [10].

There is limited information available on the nutrition knowledge and nutrition training needs of general practitioners in Australia and, to our knowledge, none is available for GP trainees in this country. Registrar members of GPRA were invited to participate in a survey which aimed specifically to assess (1) nutrition knowledge, (2) perceived role of GPs in disseminating nutrition advice, and (3) confidence in their ability to advise on prevention and treatment of cardiovascular diseases through use of evidence-based nutrition practices.

\section{Methods}

2.1. Study Sample and Survey Administration. An online nutrition survey (SurveyMonkey) was posted on the website of General Practice Registrars Australia (GPRA) registrars between February 22 and June 24, 2013, and was funded by the Almond Board of Australia. GPRA represents over 21,000 medical students and GP members at various stages of their training [10]. At any given time, approximately 6,000 are registrar members $(6,123$ at time of writing).

GP registrars have completed their basic medical training and are currently enrolled in further specialist GP training which will allow them membership of either the Australian College of Rural and Remote Medicine or the Royal Australian College of General Practitioners. Registrar members can have completed their training up to two years earlier and be working independently.

GP registrars registered with the GPRA were sent an email, inviting them to participate in the anonymous online survey. Participants completing the survey were placed in a draw to win one of five food hampers worth $\$ 100$ each. The project was approved by the Deakin University Human Ethics Advisory Group: Project number HEAG-H 87_2012.

2.2. Survey Design and Content. The survey took approximately 8-10 minutes to complete. One case study of a patient with elevated risk of cardiovascular disease was included and participants rated the appropriateness of different lifestyle strategies to address this patient's risk. Of the 15 strategies proposed, 11 were nutritional in nature and two of these (avoiding spicy foods and reducing fresh fruit intake) were deliberately included as strategies not in line with the Australian dietary guidelines, to check whether students responded appropriately to these. Two further questions related indirectly to the relevant nutrition content (the term used was "lifestyle strategies to reduce cardiovascular risk") taught and assessed during medical training, one question related to nutrition education postgraduation, one assessed attitude regarding the role of the GP as provider of nutrition information and another the self-assessment of confidence in the provision of nutrition advice, and one question each related to nutrition resources and referral practices to other health professionals. Four other questions provided consent to take part in the survey as well as information on demographic characteristics.

2.3. Statistical Analysis. The data was summarized using descriptive statistics, percentages, and graphs.

\section{Results}

3.1. Demographics. Of the approximately $6,000 \mathrm{GP}$ registrars invited to participate, a total of $93(2 \%)$ completed the online nutrition survey; four of these did not provide demographic information. Of the 89 remaining participants, $23 \%$ were male and $77 \%$ were female, and $67(75 \%)$ were aged less than 35 years. Sixty-seven percent $(n=59)$ had graduated within the last 2 to 6 years, $16 \%$ had graduated within 7-9 years, and $17 \%$ had graduated more than 10 years beforehand.

3.2. Responses to Clinical Scenario: Elevated Blood Lipids, Prehypertension, and Overweight. To gain insight into the nutrition knowledge of GP registrars, the following patient scenario was presented: "Mr. Jones, a 50-year-old mid-level manager in a large car manufacturing company, presents to you as a patient as he had a blood lipid check through a workplace screening initiative. His fasting blood lipid results are: total cholesterol $6.30 \mathrm{mmol} / \mathrm{L}(244 \mathrm{mg} / 100 \mathrm{~mL})$, LDL cholesterol $4.11 \mathrm{mmol} / \mathrm{L}(159 \mathrm{mg} / 100 \mathrm{~mL})$, HDL cholesterol $1.57 \mathrm{mmol} / \mathrm{L}(61 \mathrm{mg} / 100 \mathrm{~mL})$, and triglyceride $1.38 \mathrm{mmol} / \mathrm{L}$ $(122 \mathrm{mg} / 100 \mathrm{~mL})$. His Body Mass Index (BMI) is $28 \mathrm{~kg} / \mathrm{m}^{2}$ and seated blood pressure is $128 / 85 \mathrm{mmHg}$." To answer the question "How appropriate are the following to assist in reducing Mr. Jones' cardiovascular risk?," respondents were required to choose from a list of strategies and indicate the level of appropriateness of each one, namely, "highly appropriate," "somewhat appropriate," "not appropriate," or "do not know" (Figure 1). They were also given space to write comments in their own words.

3.3. Dietary Responses. Almost all respondents (99\%) indicated that weight loss was a "highly" (84\%) or "somewhat" (15\%) appropriate goal, and most of them (98\%) indicated that reducing total fat intake was "highly" (59\%) or "somewhat" (39\%) appropriate. Ninety-nine percent agreed that a reduction in saturated fat was an appropriate strategy, with most of them (90\%) indicating this was "highly appropriate." In terms of salt reduction, about half (51\%) indicated that "reducing salt intake" was "highly appropriate," and a further 39\% chose "somewhat appropriate," but 10\% felt salt reduction was "not appropriate." The option of reducing alcohol intake to a maximum of 2 standard drinks per day was well supported: $98 \%$ viewed this as "highly appropriate" or "somewhat appropriate." Increasing vegetable intake was rated "highly" (83\%) or "somewhat appropriate" (15\%) by $98 \%$ of respondents. Avoiding all takeaway foods was only "highly appropriate" to $22 \%$ of registrars. Fifty-one felt it to be "somewhat" appropriate, while $26 \%$ did not agree that it was appropriate at all. The suggestion that Mr. Jones 
How appropriate are the following to assist in reducing Mr. Jones's cardiovascular risk?

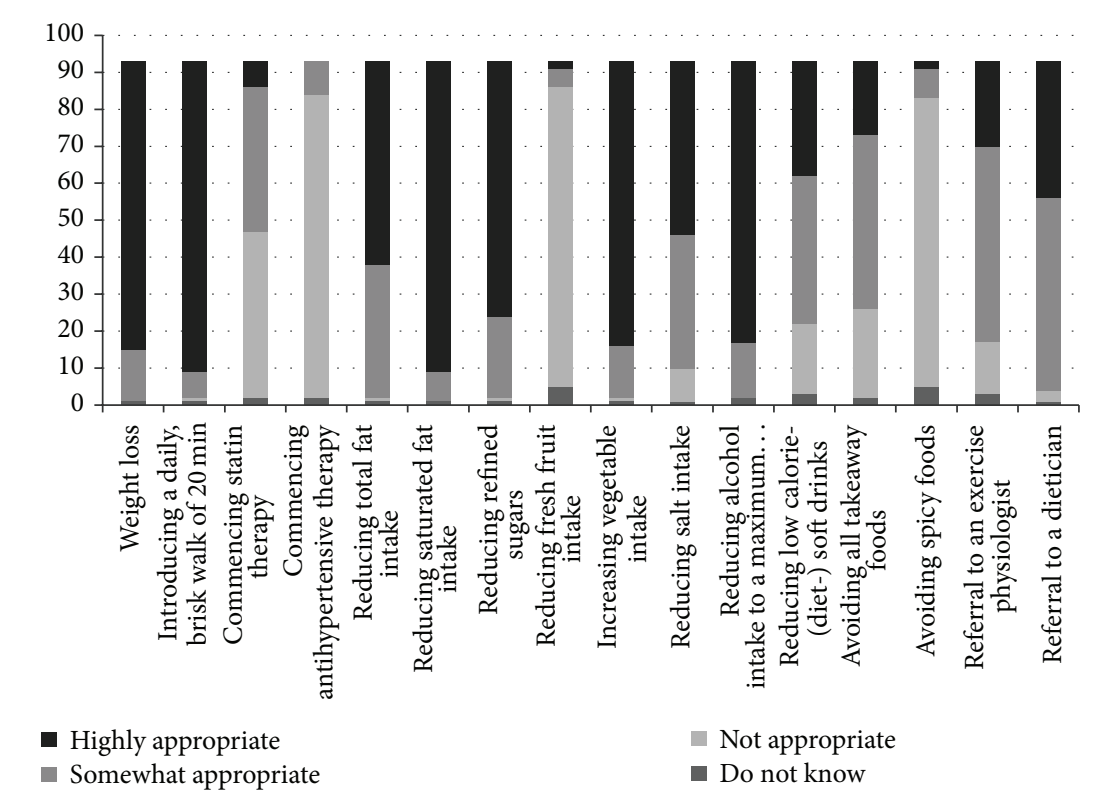

FIGURE 1: Cumulative perceived appropriateness of strategies to assist cardiovascular risk.

could be referred to a dietitian met with generally strong approval: $96 \%$ felt that this would be either "highly" (40\%) or "somewhat" (56\%) appropriate and only 3\% felt that it was "not appropriate." The appropriateness of providing recommendations related to refined sugar intake (reducing refined sugars) was less clear, with $74 \%$ indicating that this was "highly appropriate" but one-quarter (24\%) indicated that this was only "somewhat" so.

With respect to the two inappropriate strategies included as a challenge, most registrars indicated that it was inappropriate to provide advice to avoid spicy foods (84\%) with only $11 \%$ choosing this recommendation. Most registrars (87\%) ticked that a reduction in fruit intake was "not appropriate" (which is in keeping with the dietary recommendation), whilst $12 \%$ either did not know (5\%) or rated this as being appropriate to some degree (7\%). Reduction of diet-soft drinks drew a mixed response: $76 \%$ indicated that this was appropriate to some degree, but $20 \%$ indicated that this was not appropriate and 3\% did not know.

3.4. Nonnutritional Lifestyle Strategies. Almost all GP registrars $(98 \%)$ indicated that a daily 20 -minute walk would be highly $(90 \%)$ or somewhat $(8 \%)$ appropriate. A high proportion of registrars $(79 \%)$ also indicated that it would be highly $(25 \%)$ or somewhat $(57 \%)$ appropriate to refer the patients to an exercise physiologist, but $15 \%$ chose "not appropriate" and 3\% were unsure.

A free text box was also provided for respondents to answer the question "At the consultation, what specific actions or medications would you suggest that Mr. Jones takes?" Only $19 \%$ of respondents wrote that smoking status should be checked or acted on, while $31 \%$ specified "lifestyle changes" of some sort, with $90 \%$ specifically mentioning exercise.

3.5. Medication Use Suggested by GP Trainees. From the list of given strategies, using some form of statin therapy to lower the patient's cholesterol levels was indicated to be "highly appropriate" by only $8 \%$ of registrars but a further $42 \%$ chose "somewhat appropriate"; only $2 \%$ selected "not appropriate." In contrast, not a single respondent indicated that antihypertensive mediation was "highly appropriate" and only $10 \%$ selected "somewhat" so, while $88 \%$ indicated that it was "not appropriate" ( $2 \%$ were unsure). Most participants $(78 \%)$ indicated in their written comments that medication would not be their first course of action.

3.6. Professional Development Relating to Effectiveness of Lifestyle Strategies. Respondents were asked "Have you undertaken any professional development activities related to the effectiveness of lifestyle strategies to reduce cardiovascular risk since graduating from medicine?" Just over half responded in the affirmative $(n=38)(54 \%)$, whilst the remainder responded negatively $(41 \%)$ or could not recall (5\%).

3.7. Attitudes and Perceived Role of GPs in Provision of Nutrition Advice to Patients. All the registrars responded with "Yes" to the question "Do you think that a General Practitioner has a role to play in the provision of nutrition/lifestyle advice to their patients?" Space was also provided for comments, and 20 participants indicated that they felt GPs had an important role to play, in conjunction with other trained allied health professionals, but time constraints and lack of 
TABLE 1: Confidence in providing nutrition recommendations to patients.

\begin{tabular}{lc}
\hline Answer options & Response, percent $(N=93)$ \\
\hline Very confident & $15 \%$ \\
Moderately confident & $51 \%$ \\
Somewhat confident & $29 \%$ \\
Little confidence & $5 \%$ \\
Not confident at all & $0 \%$ \\
\hline
\end{tabular}

nutrition skills were cited as impediments. The attitudes of the GPRs are indicated by the following comments.

(i) "GPs have a very important role in providing this advice" (18/20 respondents expressed some version of this view).

(ii) "...I feel time is a large impediment in the GP's ability to provide complex nutritional advice to patients" (2/20 respondents expressed this view).

(iii) "Someone who lacks motivation and has no other support outside their GP is unlikely to succeed in a lifestyle change. I am frequently referring to dietitians and exercise physiologists for patient support and education" (one respondent).

(iv) "I think most medical school training needs to increase the emphasis on nutrition and addressing lifestyle risk factors" (one respondent).

(v) “... definitely a role but not one that many GPs are wellequipped to carry out specifics of" (one respondent).

3.8. Confidence in Providing Nutrition Recommendations. Respondents were asked "How confident are you in providing accurate nutrition recommendations to patients?" Most participants (95\%) felt at least "somewhat confident" in their ability to pass on accurate nutrition information to patients, with 5\% reporting "little confidence" (Table 1 ). There was no relationship between the treatment options identified correctly as "highly appropriate" and perceived confidence (data not shown).

3.9. Referral to Allied Health Professionals. In the last 12 months, $95 \%$ of registrars reported referring patients to a dietitian, $53 \%$ to an exercise physiologist, and $27 \%$ and $30 \%$ to a nutritionist and a nurse (type of nurse unspecified), respectively. Other health professionals utilized were personal trainers (16\%) as well as diabetes educators, physiotherapists, and gym classes (all less than 6\%). Only three participants indicated that they did not refer to any allied health professionals. One provided the comment that choice for referral was limited due to country location, but the same person had recently referred to a dietitian, nurse, and exercise physiologist.

3.10. Recollection of Curriculum Related to Nutrition to Reduce Cardiovascular Risk during Initial Medical Training. Respondents were asked "Were lifestyle strategies to reduce

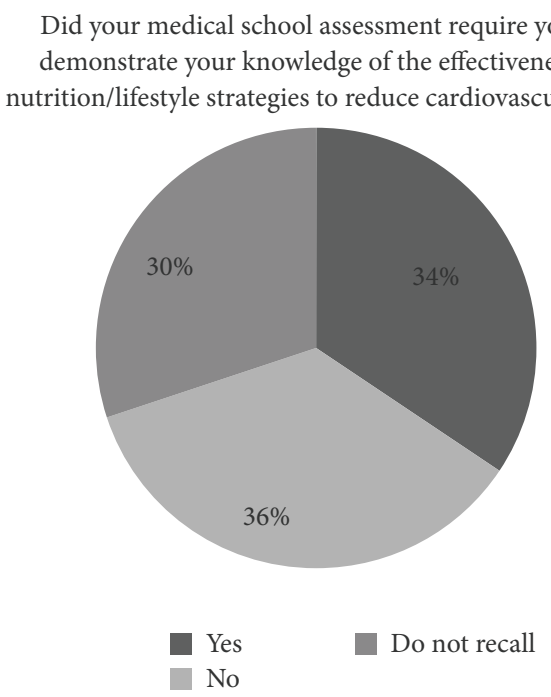

FIgURE 2: Assessment of knowledge of nutrition/lifestyle strategies to reduce cardiovascular risk by GP's medical school.

cardiovascular risk highlighted during your initial medical training?" The majority of registrars (84\%) answered "Yes" with only eight $(9 \%)$ responding "No," whilst seven $(8 \%)$ could not recall. In response to the question "Did your medical school assessment require you to demonstrate your knowledge of the effectiveness of nutrition/lifestyle strategies to reduce cardiovascular risk?", fewer registrars indicated that they were able to recall being required to demonstrate this knowledge: approximately one-third reported being required to demonstrate their knowledge but two-thirds were either not required to demonstrate nutrition knowledge or not able to recall this (Figure 2).

\section{Discussion}

In this survey, Australian GP registrars were presented with the scenario of a patient presenting after a health screening which identified prehypertension, hyperlipidemia, and overweight. It was found that the registrars' nutrition knowledge related to recommended strategies to reduce cardiovascular risk was high for the long-established strategies, including weight loss, increasing vegetables, and limiting alcohol intake. Another long-term strategy, reducing total fat, was rated as being highly appropriate by many (60\%) respondents, but more felt that reduction of saturated fat in particular was highly appropriate, in keeping with the recent recommendations by public health agencies $[11,12]$. In terms of salt reduction, about half the group indicated that salt reduction was highly appropriate but $10 \%$ thought that it was inappropriate, despite the Australian Dietary Guidelines recommendations to "Limit intake of foods and drinks containing added salt" [11] which were particularly relevant to the patient in the scenario with prehypertension. As a comparison, a survey of Australian GPs published in 2005 found that, of the 163 (45\%) who completed a questionnaire, only a minority strongly agreed that they 
would consider (37\%) or provide (22\%) nutrition counselling for hypertension [13], while five years later only $34 \%$ of patients with hypertension reported receiving advice from GPs to reduce salt intake [7].

Less than one-quarter thought that it was highly appropriate to avoid all takeaway foods and this may reflect the view that some takeaway foods may be nutritionally acceptable or that it is not necessary to avoid all takeaway foods.

In other knowledge areas, reducing refined sugar and the use of diet-soft drinks had varying responses. This was also true to some extent of the recommendation to reduce fruit intake, where the desirable response was that this was "not appropriate"; $7 \%$ of respondents suggested that this was appropriate to some extent, while $5 \%$ ticked "do not know." This may reflect confusion around these issues and indicates that GP registrars could benefit from receiving clear guidelines on these topical nutrition issues.

It was reassuring that over $95 \%$ of GP registrars indicated that they approved (either highly or somewhat) of referral of the patient to a dietitian. This could indicate that, in this group of relatively recent medical graduates, the benefits of a multidisciplinary team-based approach to patient management are supported. Such a conclusion appears to be backed by the similarly high reported rate of referral to other health professionals and aligns with the philosophy that multidisciplinary care is seen as being critical to improving the primary health care of Australia [14, 15].

There is evidence that medical graduates do not receive sufficient training in nutrition throughout the world, including Australia $[9,16,17]$. More than $80 \%$ of the registrars in the present study recollected receiving education on nutrition/lifestyle strategies to reduce cardiovascular risk, but it is of concern that only one-third recalled being asked to actually demonstrate their nutrition knowledge. In one other study of GP trainees, in Netherlands, participants agreed that a lack of nutrition training and education was a vital influence on the extent of nutrition information given to patients, the same barriers as for GPs in that country [18]. If medical schools wish to ensure that all graduates exhibit basic nutrition competencies, then it is fundamental that students are provided with assessment opportunities to demonstrate competency.

Policies in the UK clearly acknowledge the core responsibilities of doctors to address nutrition in patient care [19]. It is clear that this group of GP registrars endorses the importance that GPs play in providing basic nutrition and lifestyle advice to their patients, but time and lack of nutrition skills were cited as impediments. Most participants felt at least "somewhat confident" in their ability to pass on accurate nutrition information. These results confirm findings from a recent survey conducted in New Zealand medical students which indicated that this group has positive attitudes towards and moderate confidence in incorporating nutrition care into practice [20]. Strategies to facilitate students' confidence in providing nutrition care are warranted and it is clear that some ongoing nutrition education would be beneficial, given the mixed knowledge of the health benefits of reducing salt, the use of diet-soft drinks, and use of refined sugar.
One key limitation of this study is the low response rate and small numbers of respondents. We suggest that this small sample of GP registrars, who voluntarily completed this questionnaire, probably represents a "nutritionally aware" group not representative of the general population of Australian GP registrars. It seems likely that this select group of relatively recent medical graduates displays greater levels of nutrition knowledge and confidence compared to the general population of GP registrars. There appear to be real challenges to engaging GP registrars' interest in delivering nutrition/lifestyle support to their patients [21,22].

\section{Conclusions}

In this study, voluntary participants in a survey of GP registrars in Australia displayed an understanding of many of the key dietary recommendations for reducing cardiovascular risk, but there was a lack of consensus regarding the recommendation for reduction of salt intake. There was also a mixed level of confidence in providing nutrition advice. Appropriate nutrition education prior to graduation and postgraduation is recommended to ensure that GP registrars develop the skills and confidence to support patients in making healthy dietary choices.

\section{Conflict of Interests}

The authors declare that they have no conflict of interests regarding the publication of this paper.

\section{References}

[1] WHO/FAO, Diet, Nutrition and the Prevention of Chronic Diseases: Report of the Joint WHO/FAO Expert Consultation 2002, World Health Organization, Geneva, Switzerland, 2003.

[2] O. T. Cate, L. Snell, and C. Carraccio, "Medical competence: the interplay between individual ability and the health care environment," Medical Teacher, vol. 32, no. 8, pp. 669-675, 2010.

[3] A. S. Truswell, G. J. Hiddink, and J. Blom, "Nutrition guidance by family doctors in a changing world: problems, opportunities, and future possibilities," The American Journal of Clinical Nutrition, vol. 77, no. 4, pp. 1089S-1092S, 2003.

[4] Australian Medical Council, Assessment and Accreditation of Medical Schools: Standards and Procedures, 2009, Australian Medical Council (ACT), Canberra, Australia, 2009.

[5] F. C. Leslie and S. Thomas, "Competent to care. Are all doctors competent in nutrition?" The Proceedings of the Nutrition Society, vol. 68, no. 3, pp. 296-299, 2009.

[6] L. Ball, "Nutrition care in general practice: are we waiting for patients to ask?" Australian Family Physician, vol. 40, no. 7, p. 463, 2011.

[7] A. O. Booth and C. A. Nowson, "Patient recall of receiving lifestyle advice for overweight and hypertension from their General Practitioner," BMC Family Practice, vol. 11, article 8, 2010.

[8] M. Mowe, I. Bosaeus, H. H. Rasmussen et al., "Insufficient nutritional knowledge among health care workers?" Clinical Nutrition, vol. 27, no. 2, pp. 196-202, 2008.

[9] C. Nowson, M. Roshier-Taks, and B. Crotty, "Nutrition competencies for the prevention and treatment of disease in Australian 
medical courses," Medical Journal of Australia, vol. 197, no. 3, p. 147, 2012.

[10] GPRA, About General Practice Registrars Australia (GPRA), 2014, http://gpra.org.au/about-us/.

[11] NHMRC, Australian Dietary Guidelines: Providing the Scientific Evidence for Healthier Australian Diets, National Health and Medical Research Council, Canberra, Australia, 2013.

[12] T. B. Walker and M. J. Parker, "Lessons from the war on dietary fat," Journal of the American College of Nutrition, vol. 33, no. 4, pp. 347-351, 2014.

[13] L. Nicholas, D. Pond, and D. C. K. Roberts, "The effectiveness of nutrition counselling by Australian General Practitioners," European Journal of Clinical Nutrition, vol. 59, supplement 1, pp. S140-S146, 2005.

[14] G. K. Mitchell, J. J. Tieman, and T. M. Shelby-James, "Multidisciplinary care planning and teamwork in primary care," The Medical Journal of Australia, vol. 188, no. 8, supplement, pp. S61S64, 2008.

[15] Department of Health and Ageing, Primary Health Care Reform in Australia: Report to Support Australia's First National Primary Health Care Strategy, edited by: Department of Health and Ageing, Australian Government, Canberra, Australia, 2009.

[16] K. M. Adams, M. Kohlmeier, and S. H. Zeisel, "Nutrition education in U.S. medical schools: Latest update of a national survey," Academic Medicine, vol. 85, no. 9, pp. 1537-1542, 2010.

[17] M. Chung, V. J. van Buul, E. Wilms, N. Nellessen, and F. J. P. H. Brouns, "Nutrition education in European medical schools: results of an international survey," European Journal of Clinical Nutrition, vol. 68, pp. 844-846, 2014.

[18] H. J. S. Maiburg, G. J. Hiddink, M. A. Van't Hof, J. J. Rethans, and J. W. Van Ree, "The NECTAR-Study: development of nutrition modules for general practice vocational training; determinants of nutrition guidance practices of GP-trainees," European Journal of Clinical Nutrition, vol. 53, no. 2, pp. S83S88, 1999.

[19] General Medical Council, Tomorrow's Doctors: Outcomes and Standards for Undergraduate Medical Education, General Medical Council, London, UK, 2009.

[20] J. Crowley, L. Ball, D. Y. Han, B. Arroll, M. Leveritt, and C. Wall, "New Zealand medical students have positive attitudes and moderate confidence in providing nutrition care: a crosssectional survey," Journal of Biomedical Education. In press.

[21] R. F. Kushner, "Barriers to providing nutrition counseling by physicians: a survey of primary care practitioners," Preventive Medicine, vol. 24, no. 6, pp. 546-552, 1995.

[22] L. E. Ball, B. Desbrow, M. Yelland, and M. D. Leveritt, "Direct observation of the nutrition care practices of australian general practitioners," Journal of Primary Health Care, vol. 6, no. 2, pp. 143-147, 2014. 

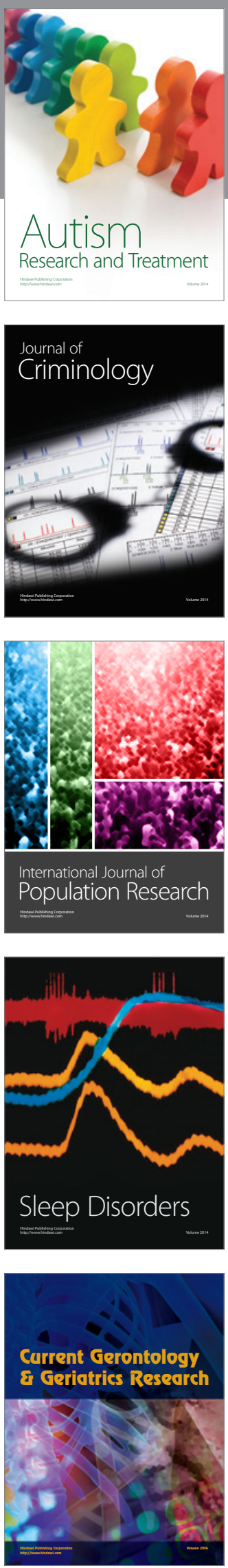
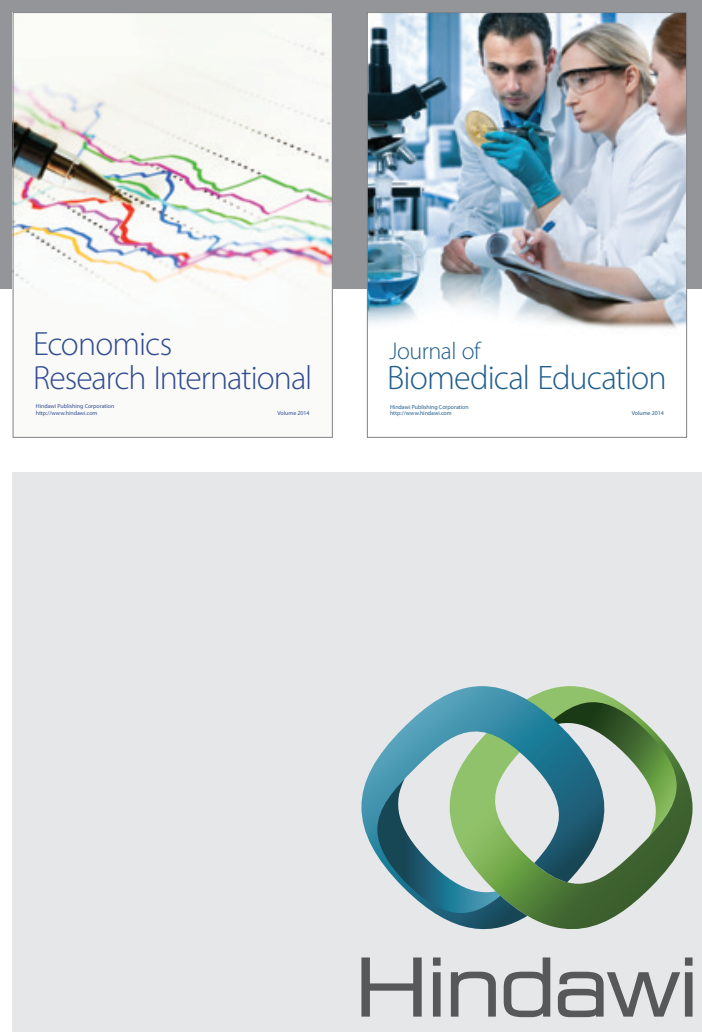

Submit your manuscripts at

http://www.hindawi.com
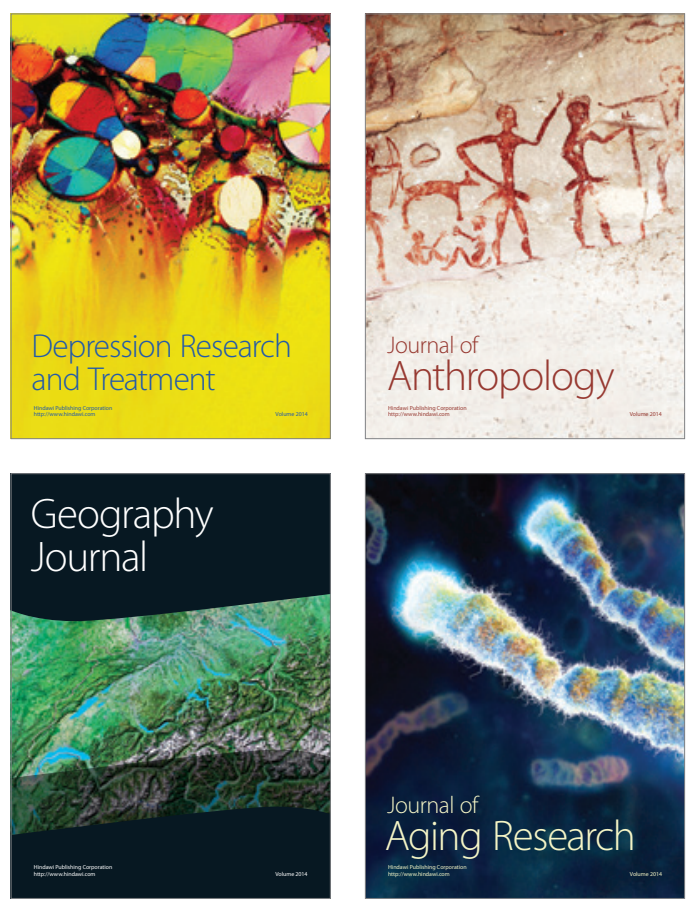
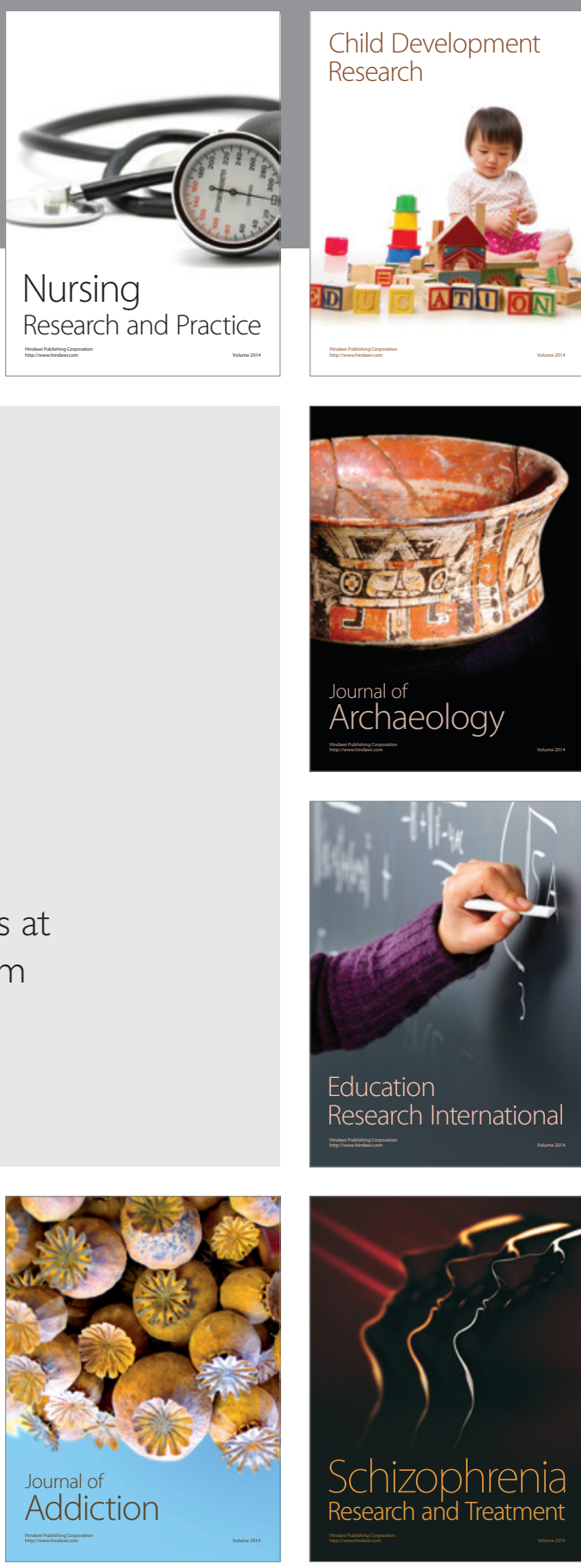

(D)
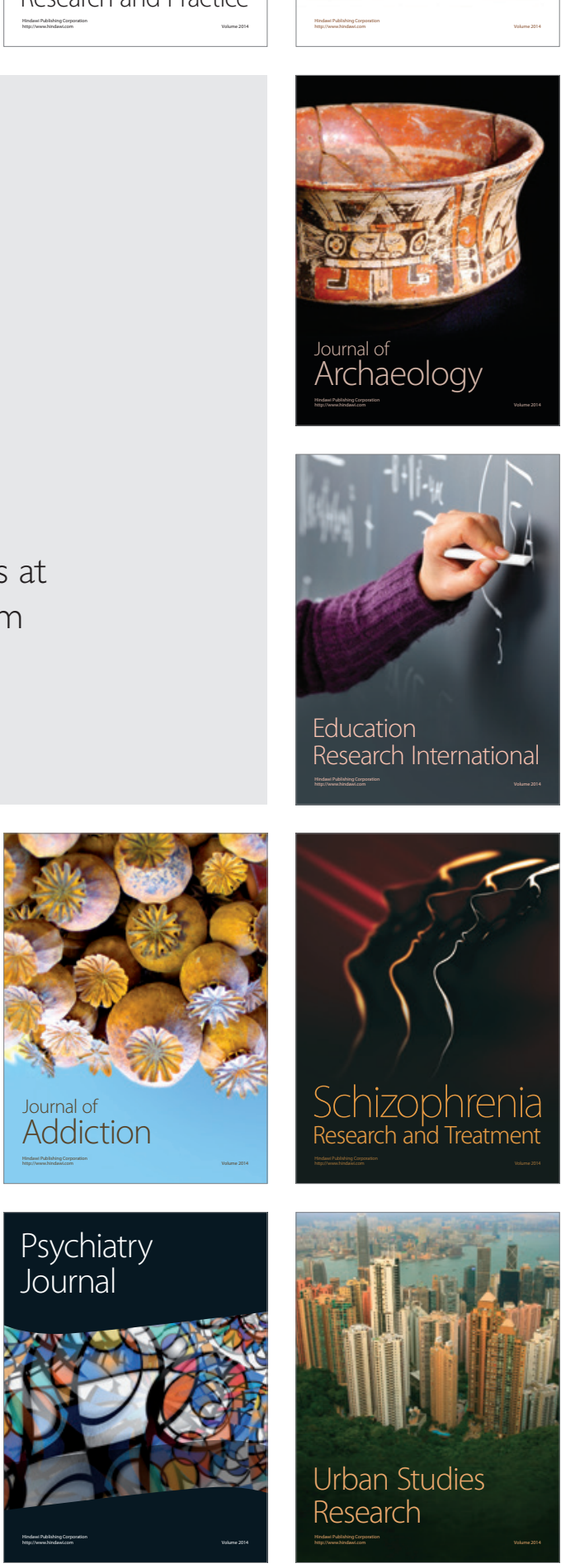